\title{
The Role of a Design Studio in a Mechanical Engineering Department
}

\section{Dr. Dustyn Roberts P.E., University of Delaware}

Dustyn Roberts received her B.S. in Mechanical and Biomedical Engineering from Carnegie Mellon University (2003), her M.S. in Biomechanics \& Movement Science (2004) from the University of Delaware, and her Ph.D. in Mechanical Engineering (2014) from New York University. She has six years of professional experience in the robotics and medical fields, and is passionate about translational research and engineering education.

\section{Prof. Jenni Buckley, University of Delaware}

Dr. Buckley is an Assistant Professor of Mechanical Engineering at University of Delaware. She received her Bachelor's of Engineering (2001) in Mechanical Engineering from the University of Delaware, and her MS (2004) and PhD (2006) in Mechanical Engineering from the University of California, Berkeley, where she worked on computational and experimental methods in spinal biomechanics. Since 2006, her research efforts have focused on the development and mechanical evaluation of medical and rehabilitation devices, particularly orthopaedic, neurosurgical, and pediatric devices. She teaches courses in design, biomechanics, and mechanics at University of Delaware and is heavily involved in K12 engineering education efforts at the local, state, and national levels. 


\section{The Role of a Design Studio in a Mechanical Engineering Department}

\section{Introduction}

Academic maker spaces, design centers, innovation institutes, and creativity labs of different kinds are becoming popular hubs of activity on many campuses - particularly within engineering colleges and departments. Some of these centers, such as Stanford's d-school and Penn State's Learning Factory [1], [2], have existed for over a decade. Others, such as Boston University's Engineering Product Innovation Center, are relatively recent developments [3], [4]. These spaces generally offer a physical location with fabrication resources and support for students to learn and work in a hands-on environment. However, they are more than just fabrication facilities: a key element of a maker space is the community itself. In other words, the people matter just as much as (or more so than) the machines. A participatory culture that encourages informal interactions between the communities the maker space serves is what distinguishes it from a facility used only for fabrication. Though each maker space has a unique purpose relative to its home institution, the spaces have all had an impact on embedding design experiences into the campus culture.

This paper describes the arrival of the Design Studio in a particular mechanical engineering department and describes its impact on students. From student-led design and construction teams through surveys of recent alumni, the methods and results of this single case study can serve as a template for development and evaluation of new maker spaces to review as they plan and implement their own spaces to foster design, innovation, and entrepreneurship skills in undergraduate engineering students.

\section{Overview of the Design Studio}

The Design Studio is a 5,500 square foot student-run space consisting of several interconnected rooms on the ground floor of a building primarily used to house the mechanical engineering department offices and labs (Figure 1). It began in 2012 with just one room that was previously being used as a large storage space, and grew strategically as departmental needs evolved. Students helped design and build the space (Figure 2, left), and the grand opening was held in March of 2014. 


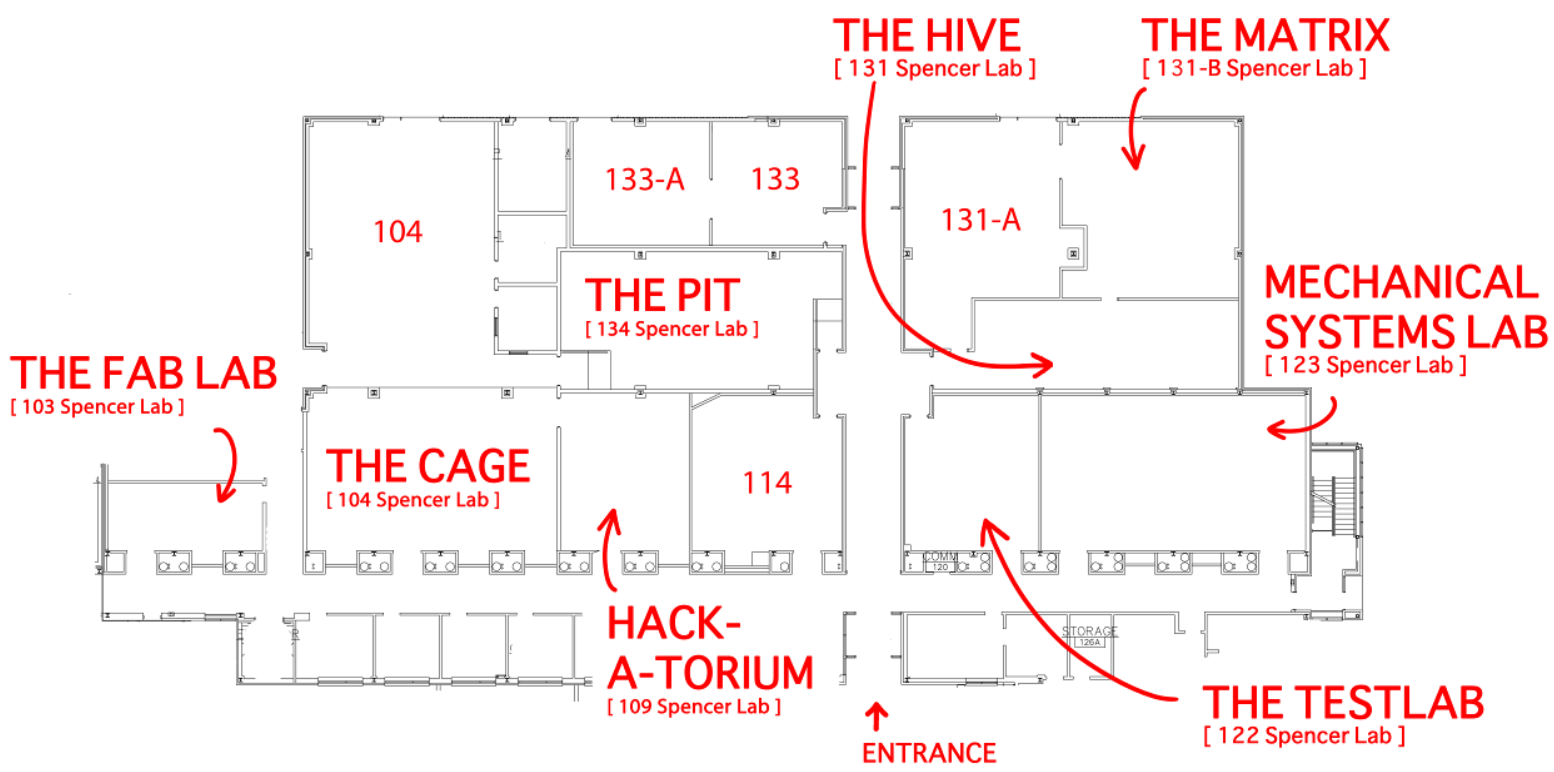

Figure 1: The layout of the Design Studio, indicated by the named rooms. The College of Engineering's student machine shop is conveniently located adjacent to the Design Studio in room 104 and encompasses part of The Cage. For reference, room 123 is approximately 47’ $\mathrm{x}$ 27 , or $1270 \mathrm{ft}^{2}$.

\subsection{Space, Equipment, and Resources}

The space and equipment are constantly growing and evolving to serve the needs of our students and department. The Fab Lab houses most of our digital fabrication equipment (three Stratasys uPrint SE Plus 3D printers), two electronics workbenches, and a variety of electronic rapid prototyping equipment including Arduino-based Sparkfun Inventor Kits and peripheral sensors. The Cage is home to all of our hand tools (e.g., portable drills, Dremels, sanding equipment, wrenches) and several benchtop tools (e.g. belt sander, drill press), as well as prototyping space. The Universal VLS4.60 laser cutter lives in the Hack-A-Torium next to a fume hood, several large work tables, and a lot of project storage bins. The Pit has room for group work and a wall lined with desktop computers, as well as two lounge areas for more casual collaborations. The Test Lab houses our sensor inventory and two large tension testing rigs, and the Mechanical Systems Lab is home to three out of four of our laboratory courses. Finally, the Hive is primarily used for teaching assistant (TA) office hours and tutoring, and the Matrix is a design lab with animal tissue storage and testing equipment controlled by the biomedical engineering department. 

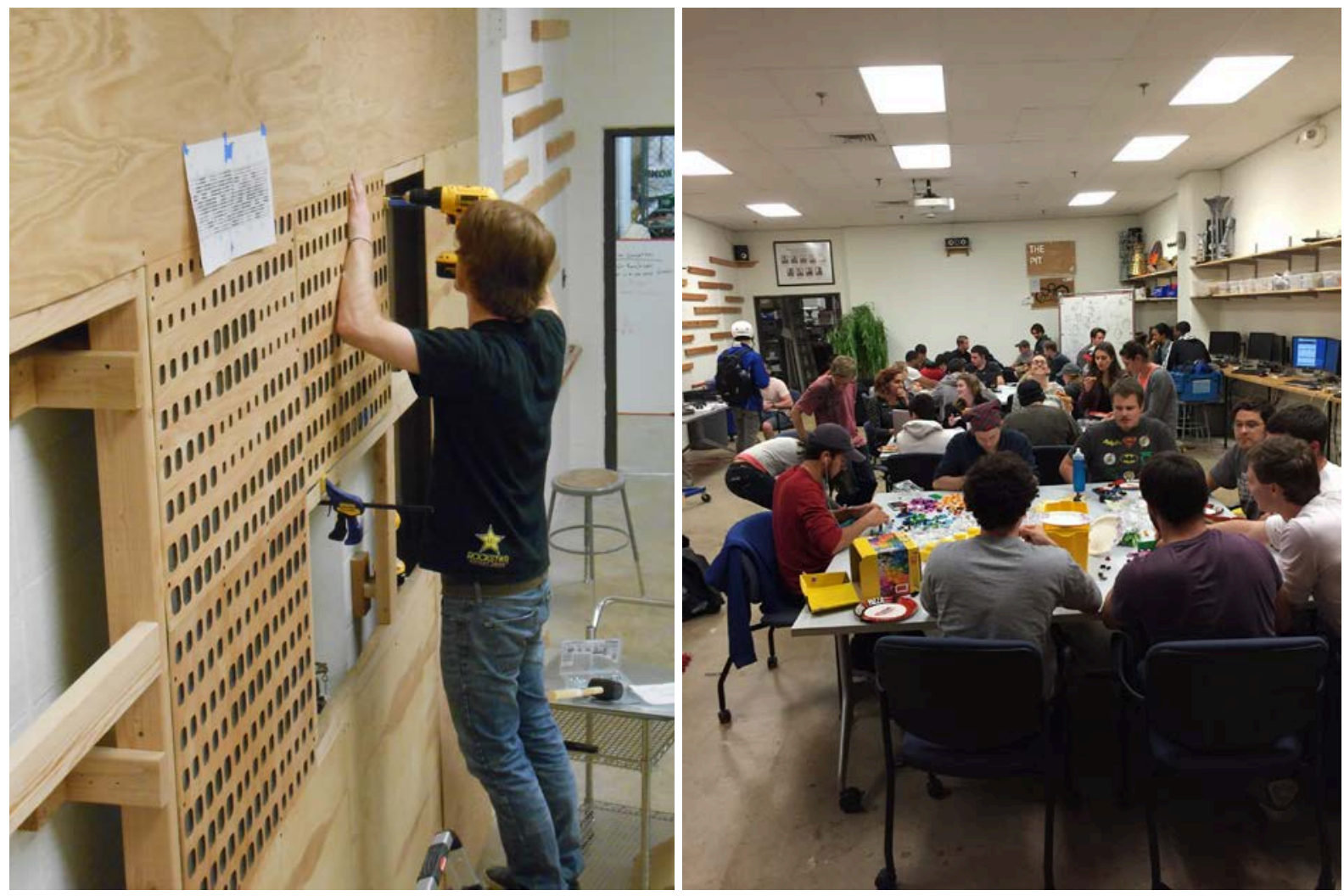

Figure 2: (Left) A student helps build out the Design Studio, (Right) The Mechanical Engineering Student Squad (MESS) hold a pizza and Legos night in the Design Studio

\subsection{Funding and Expenses}

Initial funding for the renovations came from several key alumni, as well as contributions from the mechanical engineering department and the Office of Economic Innovations and Partnerships (OEIP). The first room of the space was outfitted with tables and hand tools for just $\$ 5,000$, and the initial investment by the alumni and department came to about $\$ 80 \mathrm{k}$ along with in-kind donation of two 3D printers by OEIP. Several grants were awarded in the last year that will cover machine shop and space upgrades as well as consumables for student projects. Ongoing support will come from the mechanical engineering department (primarily through sponsorship fees from our well established senior design program) and targeted development efforts including grants and alumni donation campaigns.

\subsection{Usage}

The resources and physical space of the Design Studio are used to support a wide range of curricular, extracurricular, research, and outreach activities.

\subsubsection{Curricular Support}

The Design Studio is used extensively to support the curriculum. Several courses have required activities that make use of the resources and/or space in the Design Studio (Table 1). For example, our sophomore level Computer-Aided Engineering Design class requires student teams to 3D print components after modeling them in Solidworks. Several courses are physically held in the space, including all of the laboratory courses and two technical electives. 
Table 1: Courses directly supported by the Design Studio

\begin{tabular}{lll}
\hline Required Classes & Required Labs & Electives \\
\hline Introduction to Engineering & *Vibration and Control & *Integrated Design \\
Computer-Aided Engineering Design & *Fluids & *Applied Controls \\
Machine Design - Kinematics and Kinetics & *Thermal Fluids & *Maker Series \\
$\begin{array}{l}\text { Machine Design - Elements } \\
\text { Senior Design }\end{array}$ & \\
\hline
\end{tabular}

*Indicates courses that are physically held in the space

\subsubsection{Extracurricular Support}

The Design Studio is used by several registered student organizations, including the Society of Automotive Engineers, Engineers Without Borders, and a department level service organization called the Mechanical Engineering Student Squad (MESS) to design, build, and test their projects as well as hold events (Figure 2, right). Additionally, The Perry Initiative, a non-profit program that runs hand-on outreach programs to inspire young women to be leaders in the fields of orthopaedic surgery and engineering, makes heavy use of the space and draws volunteers from the student community. These organizations actively share the workspace throughout the year, promoting an interdisciplinary and cross-curricular exchange of ideas throughout the four undergraduate years that students typically use the space.

\subsubsection{Research and Outreach}

Many undergraduate and graduate research assistants utilize the Design Studio to support their work. We have a strong summer undergraduate research program and summer engineering internship program for high school students, and many of these students call the Design Studio home. The Design Studio is also home to past, present, and pending NSF-funded Research Experience for Teachers (RET) and Research Experience for Undergraduates (REU) programs, and allows the department to easily host first year graduate students before their alignment with a lab and advisor.

\subsection{Intellectual Property}

According to the University of Delaware Policies and Procedures Manual [5]:

"It is policy of the University that all inventions and discoveries, together with any tangible research materials, know-how and the scientific data and other records of research including any related government protections (collectively "Intellectual Property"), which are conceived or reduced to practice or developed by University faculty, staff, or students in the course of employment at the University, or result from work directly related to professional or employment responsibilities at the University, or from work carried out on University time, or at University expense, or with the substantial use of University resources, shall be the property of the University."

Since the majority of the students that use the Design Studio are undergraduates who are not considered employees of the university, and use of the Design Studio is not considered substantial use of University resources, students retain the rights to their own ideas. 
Undergraduate research assistants, graduate students, staff, and faculty who are employees of the University may be considered differently depending on the project on which they are working.

\section{Access, Staffing, and Management}

While the student machine shop is only open during normal business hours when the full time machinist is in, the Design Studio is open to students 24/7. It is primarily student run. While it is loosely managed by two co-directors that are also full time faculty, a team of $\sim 10$ undergraduate TAs do most of the day to day work in the space. At least one of the co-directors meets with the team of students weekly to discuss ongoing maintenance, space usage, upgrades, and problems. Each of the TAs is assigned a specific role within the space that ranges from managing the 3D printing queue to general organization and restock, and these roles are detailed in a responsibilities document that is reviewed with the TAs once per semester. This allows us to be very responsive, and we can immediately implement lessons learned from curricular engagement, events held in the space, etc. into our operating procedures.

Staff support is also critical. A laboratory coordinator is in charge of major safety, maintenance, and facilities-related requests. Several other department administrative staff help support purchasing efforts in the space as well as direct students to the appropriate contacts and resources.

\subsection{Safety and Training}

The student machine shop requires formal safety training and is staffed by a full time machinist with an office inside the shop. The students are required to attend an in-person basic shop safety demo, review the online shop operating procedures, and then complete the online basic shop safety quiz with a grade of $100 \%$. The students have to retake this online quiz each fall semester. As for the Design Studio, injury risk is mitigated by locking down all machines that reside permanently in The Cage area. Only TAs trained by the co-directors have keys to unlock the equipment. Students are introduced to the tools and equipment that can't be locked down throughout the curriculum. For example, the freshmen might only need to use PVC cutters and sand paper, while juniors are expected to be able to use the band saw and drill press for fabrication tasks. These tools and skills are taught to students in small groups by Design Studio TAs during class time as needed.

\section{$4 \quad$ Impact on Students}

The impact of the Design Studio on our undergraduate population was assessed as a subcategory of outcomes in our Department's standard alumni survey, which is administered approximately every five years as part of the ABET accreditation process. In fall 2015, an online survey was distributed to all alumni of the department, both graduate and undergraduate, with active email addresses $(\mathrm{N}=2301)$ via repeated emails (Constant Contact) with links to an online survey (Qualtrics). Responses were solicited for a two-week period from mid-September to early October 2015.

The survey began with questions about our current curriculum and our alumni's satisfaction with their overall level of preparation for their respective careers. This was followed by questions about self-perceptions and the importance of three core areas of emphasis for our undergraduate program: Active Learning, Professional Development, and New Technologies. Assessment items 
for the Design Studio were embedded in the Active Learning section, which included ratings of the quality and importance of undergraduate laboratory and design spaces, design projects, inclass demonstrations, and open-ended laboratory experiences. Survey items were mapped to a 4point Likert scale, with higher values corresponding to more positive outcomes. To assess the impact of the Design Studio and associated curricular changes, the survey cohort was segmented by graduation year into pre $(<2012)$ and post (2012-2015) Design Studio creation. Pre versus Post Design Studio comparisons were made using one-way ANOVA (JMP Pro v12).

\subsection{Survey Results}

The survey response rate was $6.6 \%$, with respondents representing a wide range of graduation years (2015-1945), with concentrations in 2010-2015, 1992-1995, and 1980-1982. Only undergraduate alumni who completed the survey were included for further data analysis $(\mathrm{N}=132)$.

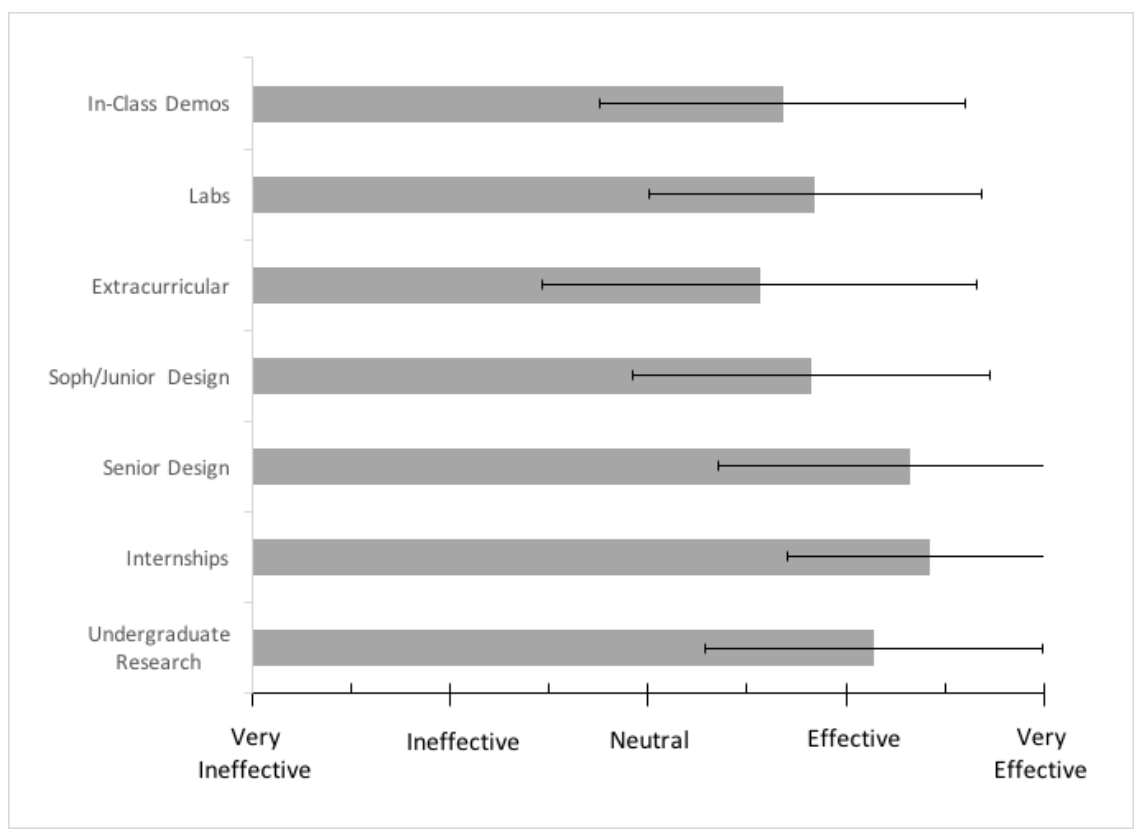

Figure 3: Results for entire survey cohort for survey questions: "Please rate the effectiveness of the following Active Learning strategies for you during your time in the UD-ME program.” Bar represents mean with whiskers \pm 1.0 standard deviation.

In general, alumni were satisfied with all elements of Active Learning in our program (Figure 3), and the creation of the Design Studio only improved the level of satisfaction (Figure 4). Comparing Pre versus Post Design Studio cohorts, Senior Design, which heavily utilizes the Design Studio resources, was ranked as significantly more effective after the creation of the Studio ( $p<0.01$, Figure 4). Similarly, there was a significant increase in effectiveness for "In Class Demos" $(p=0.03)$ and “Sophomore/Junior Design” experiences $(p=0.04)$. 


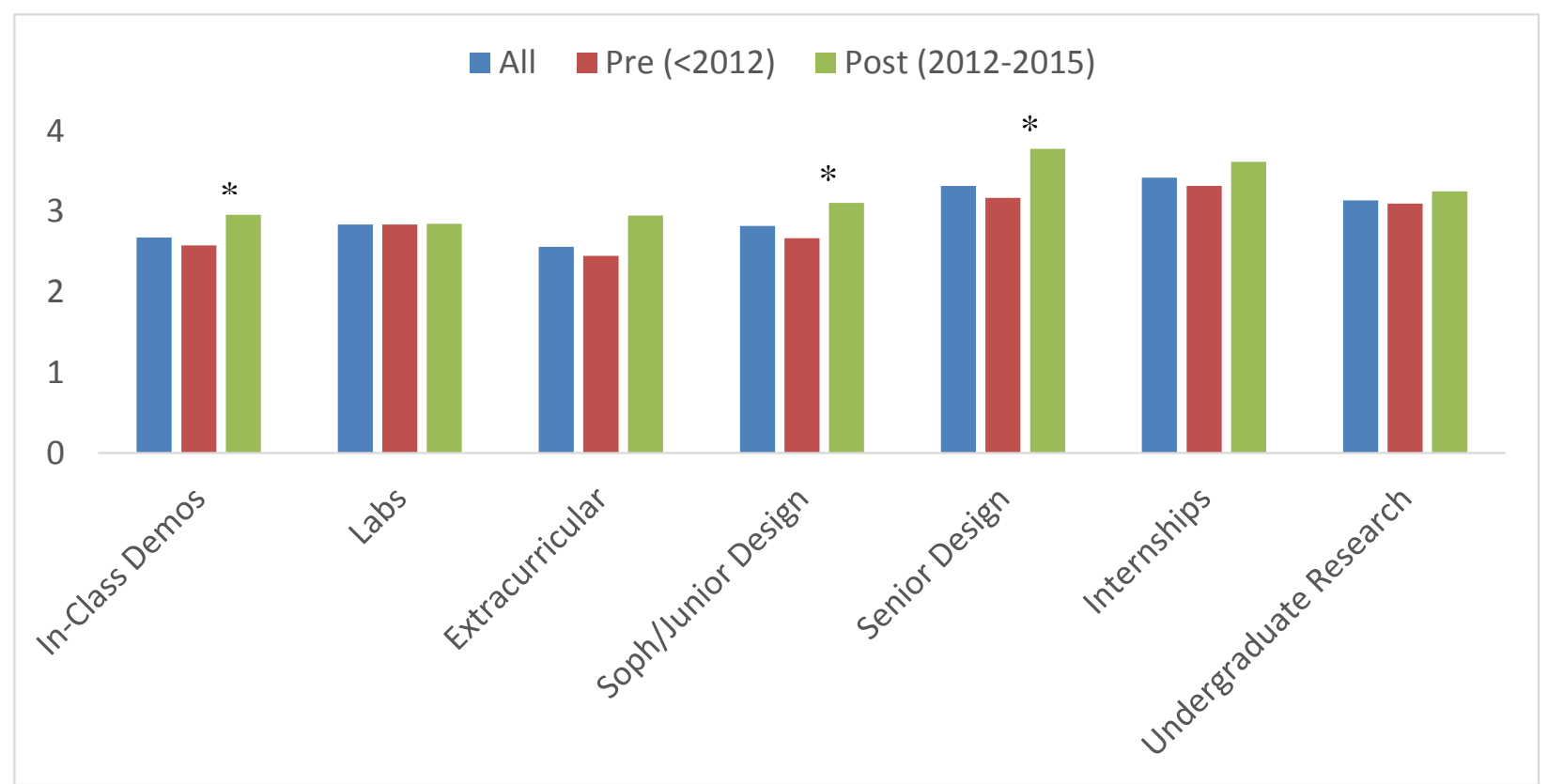

Figure 4: Results for survey question related to Active Learning: "Please rate the effectiveness of the following Active Learning strategies for you during your time in the UD-ME program." Results scored as 4-pt Likert with 4=Very Effective and 0=Very Ineffective. Results are presented for the entire survey population (All) as well as pre (<2012) and post (2012-2015) Design Studio. Asterisks indicate areas of significant pre/post changes $(\mathrm{p}<0.05)$.

\section{Conclusion and Future Work}

Although the Design Studio is a work in progress, we are happy with the results we have been able to measure so far. Aside from ongoing facilities and equipment maintenance, there are several near term changes we are exploring. A facilities upgrade will allow student ID card swipe access to all rooms, so we are looking at implementing a membership structure to the Design Studio such that access is limited by the types of training a student has taken. We are also looking for ways to make staffing more predictable to manage student demand for training on particular equipment ranging from sewing machines to power tools. In order to have a staff that can help address this, an application system was setup to identify future TAs that have either existing experience or the propensity to learn particular skills. Furthermore, as we integrate more hands on work into our curriculum, we will need to carefully balance the usage of the space for curricular, extracurricular, and research and outreach uses.

We plan to continue to evolve along with student demands and share best practices with other universities with similar spaces. Additionally, we are currently working with colleagues in our school of education to determine better assessment and evaluation techniques to help drive future changes to the space.

\section{Acknowledgements}

The authors would like to acknowledge the support of Dr. Suresh Advani, George W. Laird Professor and Chair of Mechanical Engineering, for the support of the work that led to this publication. 


\section{References}

[1] J. S. Lamancusa, J. L. Zayas, A. L. Soyster, L. Morell, and J. Jorgensen, "The Learning Factory: IndustryPartnered Active Learning,” Journal of Engineering Education, vol. 97, no. 1, pp. 5-11, Jan. 2008.

[2] J. S. Lamancusa, J. E. Jorgensen, and J. L. Zayas-Castro, "The learning factory - a new approach to integrating design and manufacturing into the engineering curriculum,” Journal of Engineering Education, pp. 103112, Apr. 1997.

[3] V. Wilczynski, C. S. O’Hern, and E. R. Dufresne, "Using an engineering design center to infuse design experience into a mechanical engineering program," in Proc. ASEE Annual Conference \& Exposition, Indianapolis, IN, 2014.

[4] C. R. Forest, R. A. Moore, A. S. Jariwala, B. B. Fasse, J. Linsey, W. Newstetter, P. Ngo, and C. Quitero, "The invention studio: A university maker space and culture," Advances in Engineering Education, vol. 4, no. 2, pp. 1-32, 2014.

[5] "Intellectual Property Protection, Ownership, and Commercialization.” [Online]. Available:

http://www.udel.edu/ExecVP/policies/research/6-06.html. [Accessed: 11-Jan-2016]. 